published in: J. Phys. A: Math. Theor. 42, 362001 (2009)

\title{
Emergence of pointer states in a non-perturbative environment
}

\author{
Marc Busse and Klaus Hornberger \\ Arnold Sommerfeld Center for Theoretical Physics, \\ Ludwig-Maximilians-Universität München, \\ Theresienstraße 37, 80333 Munich, Germany
}

\begin{abstract}
We show that the pointer basis distinguished by collisional decoherence consists of exponentially localized, solitonic wave packets. Based on the orthogonal unraveling of the quantum master equation, we characterize their formation and dynamics, and we demonstrate that the statistical weights arising from an initial superposition state are given by the required projection. Since the spatial width of the pointer states can be obtained by accounting for the gas environment in a microscopically realistic fashion, one may thus calculate the coherence length of a strongly interacting gas.
\end{abstract}

PACS numbers: 03.65.Yz, 47.45.Ab, 03.65.Ta, 02.70.Ss, 03.75.-b

\section{Introduction}

If a quantum system is brought into contact with an environment, the states forming its Hilbert space are no longer equals among each other. Rather, a particular set of wave functions, the pointer states, is singled out by the environmental interaction [1. These states are distinguished by the fact that they retain their purity for a relatively long time, say, under a Markovian master equation describing the environmental influence, while superpositions of different pointer states decay rapidly into a mixture. The associated decoherence rate can be many orders of magnitude faster than any other relevant time scale, including that of the dissipative effects induced by the environmental coupling [2, 3].

The concept of pointer states plays a fundamental role in explaining both the emergence of classicality and the operation of quantum measurement devices within the framework of quantum theory [1, 2, 3. In practice, knowing the pointer states and their time evolution allows one to directly specify the dynamics of an arbitrary initial state after the decoherence time, without solving the master equation. It is thus important to understand the emergence of pointer states in microscopically realistic environments, to characterize their form and dynamics, and to show that they constitute a basis of the system Hilbert space.

Several strategies have been proposed to determine the pointer basis. Sorting all pure states according to their linear entropy production rate allows one to select the states with minimal loss of purity [4. Another idea considers the solitonic solutions of a nonlinear equation related to the open quantum dynamics [5, 6, 7, 8, Both approaches were shown to be largely equivalent if a particle is linearly coupled to a bath of harmonic oscillators [7. The pointer states are Gaussian wave packets in these linear models [5, 6, 7, 8, and the complete, finite time decoherence in the Gaussian basis can be proved rigorously for a free particle [9]. 
The linear coupling of the system coordinate to the bath assumed in these studies is often justified on the time scale of dissipation. However, it may misrepresent the decoherence process since it implies that the decoherence rate grows above all bounds for increasingly extended quantum states. This lack of saturation of the decoherence rate is unphysical for local interactions (an artifact of the unboundedness of the position operator); it predicts rates, e.g. for collisional decoherence measurements [10], that are too large by many orders of magnitude. Moreover, previous studies did not demonstrate the conceptually important requirement that the statistical weight of an evolving pointer state is given by its initial overlap with the initial quantum state.

In this paper, we study the emergence of pointer states due to collisional decoherence, allowing for a realistic and non-perturbative description of the impact of a gas environment [11, 12. We demonstrate how the orthogonal unraveling of the master equation [13] naturally explains the formation of (non-Gaussian) pointer states and their ensuing classical evolution, as well as the appearance of the expected statistical weights. Finally, by relating the widths of the pointer states to the microscopically defined localization rate of the master equation we can estimate the coherence length in an interacting thermal gas.

\section{The general pointer basis}

To define the notion of pointer states more precisely, consider a quantum system described by a Lindblad master equation $\partial_{t} \rho=\mathcal{L}(\rho)$ due to its contact with an environment. One may say that $\mathcal{L}$ generates a pointer basis if the dynamics exhibits a separation of time scales, characterized by a decoherence time $t_{\mathrm{dec}}$, such that there exists a unique set of pure states $\mathbf{P}_{\alpha}=\left|\pi_{\alpha}\right\rangle\left\langle\pi_{\alpha}\right|$, which are independent of $\rho_{0}$ and which have the property that at all later times any initial state $\rho_{0}$ can be represented by a mixture of these states

$$
e^{\mathcal{L} t} \rho_{0} \simeq \int \mathrm{d} \alpha \operatorname{Prob}\left(\alpha \mid \rho_{0}\right) \mathrm{P}_{\alpha}(t), \quad \text { if } t \gg t_{\mathrm{dec}} .
$$

Crucially for interpreting the $\mathrm{P}_{\alpha}$ as proper pointer states, whenever $\rho_{0}$ is a superposition of mutually orthogonal pointer states $\mathrm{P}_{\beta}$, the ensuing probability distribution $\operatorname{Prob}\left(\alpha \mid \rho_{0}\right)=\sum_{\beta} w_{\beta} \delta(\alpha-\beta)$ is determined by the initial overlaps

$$
w_{\beta}=\operatorname{Tr}\left(\rho_{0} \mathrm{P}_{\beta}(0)\right) \text {. }
$$

$\mathrm{P}_{\alpha}$ evolve in time, slowly compared to the decoherence scale $t_{\mathrm{dec}}$, and they initially form a basis (usually overcomplete) with $\mathrm{d} \alpha$ being the associated measure, $\int \mathrm{d} \alpha \mathrm{P}_{\alpha}=\mathrm{I}$. The uniqueness of the evolving pointer states $\mathrm{P}_{\alpha}$ is a result of equation (11) applying to times $t$, which (while exceeding the decoherence time) can be much shorter than the above-mentioned time scale of dissipation, $t_{\mathrm{dec}} \ll t \ll t_{\mathrm{diss}}$.

Below, we demonstrate that a viable way of obtaining the $\mathrm{P}_{\alpha}$ and their time evolution is to follow the approach suggested in [5, 6, 7, 8] of using the solitonic solutions of a nonlinear dynamic equation in the space of pure states,

$$
\partial_{t} \mathrm{P}=[\mathrm{P},[\mathrm{P}, \mathcal{L}(\mathrm{P})]] \text {. }
$$

This equation is satisfied by the pure state solutions of $\partial_{t} \rho=\mathcal{L}(\rho)$, if there are any, while its nonlinear character is essential to distinguish pointer states from their superpositions. It has a natural interpretation as the deterministic part of a specific class of unravelings of the master equation, as explained below. 


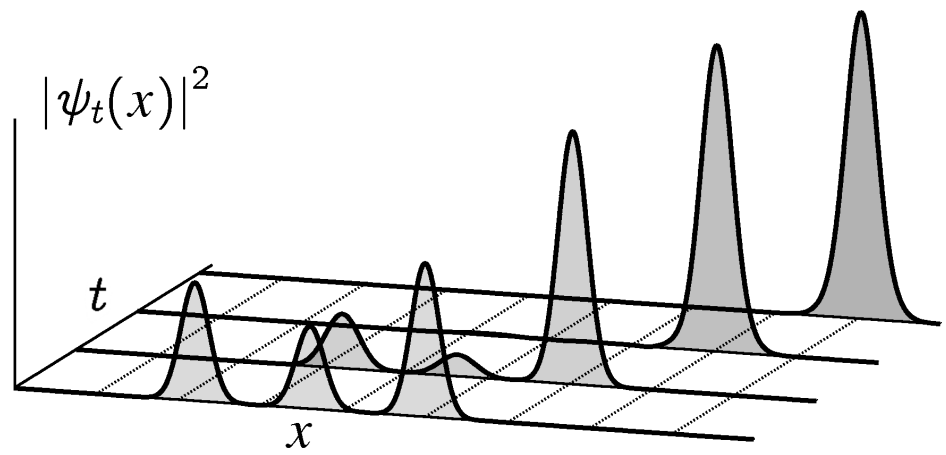

Figure 1. The nonlinear equation (7) drives any initial state $\left|\psi_{0}\right\rangle$ (here a superposition of wave packets travelling to the right) into a localized soliton $\left|\pi_{t}\right\rangle$ that moves with fixed envelope and constant velocity. These solitonic solutions form an overcomplete set, the pointer basis of collisional decoherence.

Having a solution $\mathrm{P}_{t}$ to (3) at hand, one may generate further solutions if the system has an underlying symmetry. In particular, if $\mathcal{L}$ has the form $\mathcal{L}(\rho)=$ $1 /(i \hbar)[\mathrm{H}, \rho]+\mathcal{D}(\rho)$ and $\mathbf{U}_{t}$ is a family of unitary operators satisfying

$$
\begin{aligned}
\mathrm{U} \mathcal{D}(\rho) \mathrm{U}^{\dagger} & =\mathcal{D}\left(\mathrm{U} \rho \mathrm{U}^{\dagger}\right), \\
i \hbar \partial_{t} \mathrm{U} & =[\mathrm{H}, \mathrm{U}],
\end{aligned}
$$

then $\mathrm{U}_{t} \mathrm{P}_{t} \mathrm{U}_{t}^{\dagger}$ can be shown to solve (3). This will allow us to prove that the pointer states form a basis, rendering a single pointer state sufficient to construct the whole set.

\section{Collisional decoherence}

We now apply these ideas to the 1D motion of a free particle subject to collisional decoherence [11, 12, 14. The corresponding master equation is the short time version of the full, non-perturbative, Markovian description of a test particle in a gas [15]. As such, it describes the loss of coherence but no dissipation effects, and it has the form $\mathcal{L}(\rho)=(2 m i \hbar)^{-1}\left[\mathbf{p}^{2}, \rho\right]+\mathcal{D}(\rho)$, with incoherent part

$$
\mathcal{D}(\rho)=\gamma \int_{-\infty}^{\infty} \mathrm{d} q G(q) e^{i q \times / \hbar} \rho e^{-i q \times / \hbar}-\gamma \rho .
$$

It involves the collision rate $\gamma$ and the normalized momentum transfer distribution $G(q)$, each defined by the scattering cross section and the gas temperature [11, 12, and has Lindblad structure with bounded jump operators $\mathrm{L}_{q}=\sqrt{\gamma G(q)} e^{i q \times / \hbar}$. If one disregarded the coherent part, an exponential decay of the position off-diagonal elements would ensue, $\left\langle x\left|\rho_{t}\right| x^{\prime}\right\rangle=\exp \left[-F\left(x-x^{\prime}\right) t\right]\left\langle x\left|\rho_{0}\right| x^{\prime}\right\rangle$, with a localization rate $F(s)=\gamma-\gamma \int \mathrm{d} q G(q) \exp (i q s / \hbar)$, which saturates at $\gamma$ for $s \rightarrow \infty$.

The form of $\mathcal{L}$ turns the vector representation of (3) into a nonlinear integrodifferential equation

$$
\begin{aligned}
& \partial_{t} \psi_{t}(x)=-\frac{\hbar}{2 m i} \partial_{x}^{2} \psi_{t}(x)-\psi_{t}(x) \Lambda\left[\left|\psi_{t}\right|^{2}\right](x), \\
& \Lambda[g](x)=g * F(x)-\int_{-\infty}^{\infty} \mathrm{d} y g(y) g * F(y),
\end{aligned}
$$


involving convolutions $g * F(x):=\int \mathrm{d} y g(y) F(x-y)$. Since the dispersion caused by the first term in (7) competes with the localizing effect of the second term, all solutions of (7) turn into stable solitons, $\left|\psi_{t}\right\rangle \rightarrow\left|\pi_{t}\right\rangle$ for $t \gg t_{\mathrm{dec}}$. These move with constant shape and velocity, $\left|\pi_{t+\tau}(x)\right|=\left|\pi_{t}\left(x-v_{0} \tau\right)\right|$, as demonstrated numerically in figure 1. Below, we show that these solitonic solutions can be identified with the pointer states of collisional decoherence.

\section{Soliton basis}

Even though the localization rate $F(x)$ remains bounded for large $x$, the solitonic solutions to (77) are exponentially localized, $\pi_{t}(x) \sim e^{-k\left|x-v_{0} t\right|} e^{i \varphi\left(x-v_{0} t, t\right)}$ as $x \rightarrow \pm \infty$, with $k>0$. This follows analytically by noting that for large $x$ Eq. (7) takes the asymptotic form

$$
\partial_{t} \psi_{t}(x) \sim-\frac{\hbar}{2 m i} \partial_{x}^{2} \psi_{t}(x)-\left(\gamma-a_{\psi}\right) \psi_{t}(x), \quad \text { as }|x| \rightarrow \infty,
$$

with a constant $a_{\psi}=\int_{-\infty}^{\infty} \mathrm{d} y\left|\psi_{t}(y)\right|^{2}\left|\psi_{t}\right|^{2} * F(y)$ satisfying $0<a_{\psi}<\gamma$. Here, we have used that the convolution $|\psi|^{2} * \tilde{G}(x)$ vanishes for $|x| \rightarrow \infty$, where $\tilde{G}(x)$ denotes the Fourier transform of $G(q)$. Any solitonic solution $\pi_{t}(x)=f\left(x-v_{0} t\right) e^{i \varphi\left(x-v_{0} t, t\right)}$ thus takes the form $f(x)=e^{-k|x|}$ with $k>0$ and a phase $\varphi(x, t)$ asymptotically linear in $x$.

Once a particular soliton $\mathrm{P}=|\pi\rangle\langle\pi|$ has been found, further solitonic solutions to (7) are obtained by means of Galilei transformations. The phase space translation operator $\mathrm{U}_{t} \equiv \mathrm{T}_{s, u}=\exp \left(i\left(u_{t} \mathrm{x}-s_{t} \mathrm{p}\right) / \hbar\right)$ satisfies condition (4) with (6). Moreover, condition (5) is met with $\mathrm{H}=\mathrm{p}^{2} /(2 m)$ provided $s_{t}=u_{0} t / m+s_{0}$ and $u_{t}=u_{0}$, rendering $\mathrm{T}_{s, u} \mathrm{PT}_{s, u}^{\dagger}$ also a solitonic solution of (7). This family of states, parametrized by $\Gamma=\left(s_{0}, u_{0}\right)$, forms an overcomplete basis since the identity, like any HilbertSchmidt operator, can be represented as $\mathbf{I}=\int \mathrm{d} \Gamma f(\Gamma) \mathrm{T}_{s, u} \mathrm{Q} \mathrm{T}_{s, u}^{\dagger}$ if $\mathrm{Q}$ is a trace-class operator [16].

\section{Orthogonal unraveling}

To identify the solitons as pointer states we now employ the method of quantum trajectories [13, 17, 18. In this framework, a pure initial state $P_{0}$ is propagated by a stochastic differential equation to generate an ensemble of pure states $\left\{\mathrm{P}_{t}^{i}\right\}_{i}$, whose average yields the solution of the master equation, $\mathbb{E}\left[\mathrm{P}_{t}^{i}\right]=\mathrm{e}^{\mathcal{L} t} \mathrm{P}_{0}$. Since there are infinitely many different stochastic processes with this property (unravelings), the ensemble corresponding to a fixed initial state has no observable consequences, apart form this average.

However, Eq. (10) is a statement about the solutions for all initial states, such that the physically distinguished basis in (1) may be related to a specific unraveling. In particular, a preference of a basis is obtained if the deterministic part of the unraveling exhibits solitonic solutions, such that the stochastic part no longer affects a trajectory once it has reached these solitons.

We will now show, for the case of collisional decoherence, that this behavior is found with the orthogonal unraveling $[13]$. Here, the wave function evolution has a deterministic part given by the nonlinear equation (3), which is interrupted by random

$\ddagger$ Rather than the original version $[13$, we use here the simpler orthogonal unraveling mentioned in [20. It has the advantage that the jump operators are given explicitly. 
jump\&S. The latter occur with the rate $r_{q}=\left\langle\mathrm{L}_{q}^{\dagger} \mathrm{L}_{q}\right\rangle-\left\langle\mathrm{L}_{q}^{\dagger}\right\rangle\left\langle\mathrm{L}_{q}\right\rangle$ and are effected by the (nonlinear) operators $\mathrm{J}_{q}=\left(\mathrm{L}_{q}-\left\langle\mathrm{L}_{q}\right\rangle\right) / \sqrt{r_{q}}$.

First, we consider only the deterministic part of the quantum trajectory evolution, given by (77). We will restrict the discussion to pure initial states $\left|\psi_{0}\right\rangle=\sum_{i=1}^{N} c_{i}\left|\phi_{i}\right\rangle$, which are superpositions of narrow wave packets, with variance $\operatorname{Var}_{\phi_{i}}(\mathrm{x})<2 \pi \hbar^{2} / \operatorname{Var}_{G}(q)$, situated on non-overlapping domains, $\phi_{i}(x) \phi_{j \neq i}^{*}(x)=0$, with separation $\Delta x$ sufficiently large such that $F(\Delta x) \simeq F(\infty) \equiv \gamma$. Under this assumption, which will be justified at the end of the following section, one can reformulate the nonlinear evolution (7) such that the essential part of the dynamics is already covered by the coefficients $c_{i}(t)$, obeying a closed system of equations,

$$
\frac{\mathrm{d}}{\mathrm{d} t}\left|c_{i}\right|^{2}=-2 \gamma\left(\sum_{j=1}^{N}\left|c_{j}\right|^{4}-\left|c_{i}\right|^{2}\right)\left|c_{i}\right|^{2} .
$$

This equation was studied in [19] in the context of a model for state vector reduction. As shown there, all its stable fixed points have the form $\left|c_{i}\right|=\delta_{i, n}$, and a distinguished fixed point is approached monotonically, $\left|c_{i}(t \rightarrow \infty)\right|=\delta_{i, m}$, with $m=\operatorname{argmax}_{i}\left(\left|c_{i}\right|\right)$ the index of the the largest wave packet contribution to $\left|\psi_{0}\right\rangle$.

The dynamics of the wave packets $\left|\phi_{i}\right\rangle$ is described by

$$
\begin{aligned}
\partial_{t} \phi_{i}(x)= & -\frac{\hbar}{2 m i} \partial_{x}^{2} \phi_{i}(x)-\phi_{i}(x) \Lambda\left[\left|\phi_{i}\right|^{2}\right](x) \\
& +\phi_{i}(x) \sum_{j=1, j \neq i}^{N}\left|c_{j}\right|^{2} \tilde{\gamma}_{i j}(x)
\end{aligned}
$$

with $\Lambda$ defined in (8) and a rate

$$
\tilde{\gamma}_{i j}(x)=\left|\phi_{i}\right|^{2} * F(x)-\left|\phi_{j}\right|^{2} * F(x)+\gamma,
$$

which is of the order of $\gamma$. In order to verify (10) and (11), one takes the derivative of $\psi_{t}(x)=\sum_{i} c_{i}(t) \phi_{i}(t)$ with respect to time; using

$$
\int \mathrm{d} y\left|\phi_{i}(y)\right|^{2}\left|\phi_{j}\right|^{2} * F(y)=\gamma\left(1-\delta_{i j}\right)
$$

which follows from the above assumptions on $\phi_{i}(x)$, one finds that $\psi_{t}(x)$ evolves according to (77).

Since the coefficients $c_{j \neq m}$ tend to zero according to (10), the coupling term vanishes after some time in Eq. (11), turning the latter into the soliton equation (7). In the absence of jumps, the initial superposition state $\left|\psi_{0}\right\rangle$ thus evolves into the soliton which is associated to $\left|\phi_{m}\right\rangle$, the wave packet contributing largest to $\left|\psi_{0}\right\rangle$ (see Fig. 1).

Next, we consider the probabilistic part of the quantum trajectories. Using the above assumption of localized wave packets, $\operatorname{Var}_{\phi_{i}}(\mathrm{x})<2 \pi \hbar^{2} / \operatorname{Var}_{G}(q)$, one can approximate $\int \mathrm{d} x e^{i q x / \hbar}\left|\phi_{i}(x)\right|^{2} \simeq e^{i q x_{i} / \hbar}$ with $x_{i}=\langle\mathrm{x}\rangle_{\phi_{i}}$. Under this assumption, the jump operator $J_{q}$ affects only the coefficients. They are transformed as

$$
c_{k}^{\prime}(q)=\frac{1}{\mathcal{N}}\left(e^{i q x_{k} / \hbar}-\sum_{j=1}^{N}\left|c_{j}\right|^{2} e^{i q x_{j} / \hbar}\right) c_{k},
$$

$\S$ We note that Eq. (3) appears also in the context of a diffusive (i.e. not piecewise-deterministic) unraveling. It was used in [7] to study pointer states within a linear coupling model. 
with $\mathcal{N}$ being the normalization. The jump rate takes the form

$$
r_{q}=\gamma G(q)\left(1-\sum_{j, k=1}^{N}\left|c_{j}\right|^{2}\left|c_{k}\right|^{2} e^{i q\left(x_{j}-x_{k}\right) / \hbar}\right) .
$$

It vanishes for $\left|c_{i}\right|=\delta_{i, n}$, that is, when a stable fixed point of (10) is approached. The associated wave function can then evolve into a soliton state without being further perturbed by jumps. This confirms that the quantum trajectories of the orthogonal unraveling evolve into pointer states, the solitonic solutions to (7).

Concerning the statistical weights, we now show how the stochastic process, described by (10), (14), (15) can be treated analytically for $N=2$. The average effect of the jump $\left\langle c_{k}^{\prime}\right\rangle_{G}=\int \mathrm{d} q G(q) c_{k}^{\prime}(q)$ simply interchanges the value of the coefficients,

i.e., $\left|\left\langle c_{1}^{\prime}\right\rangle_{G}\right|^{2}=\left|c_{2}\right|^{2}$. The probability of a trajectory starting from $\left|c_{1}(0)\right|^{2}<1 / 2$ to converge to $\left|c_{i}\right|=\delta_{i, 1}$ is therefore equal to the probability for an odd number of jumps. It is given by $\left(1-e^{-2 \mu(t)}\right) / 2$ for this inhomogeneous Poisson process, with $\mu(t)=\int_{0}^{t} \mathrm{~d} \tau r_{\text {tot }}(\tau)$ the integrated jump rate. In our case,

$$
r_{\text {tot }} \equiv \int \mathrm{d} q r_{q}=2 \gamma\left|c_{1}\right|^{2}\left(1-\left|c_{1}\right|^{2}\right)
$$

where $c_{1}(t)$ is a solution to (10). Rewriting (10) as

$$
2 \gamma\left|c_{1}\right|^{2}\left(1-\left|c_{1}\right|^{2}\right)=\frac{1}{2} \partial_{t} \ln \left(1-2\left|c_{1}\right|^{2}\right),
$$

we find that $\mu(t \rightarrow \infty)=-\ln \left(1-2\left|c_{1}(0)\right|^{2}\right) / 2$. The probability for an odd number of jumps is therefore $\left|c_{1}(0)\right|^{2}=\left|\left\langle\psi_{0} \mid \phi_{1}\right\rangle\right|^{2}$. If $\left|\phi_{i}\right\rangle=\left|\pi_{i}\right\rangle$ this demonstrates that the statistical weights of the pointer states in the decohered mixture is given by the overlap $\left|\left\langle\psi_{0} \mid \pi_{1}\right\rangle\right|^{2}$, in accordance with (2).

The generalization of this result to $N>2$ can be verified numerically. Taking $G(q)$ to be a Gaussian with variance $\sigma_{G}^{2}$, we have implemented the stochastic process (10), (14), (15) for various $2 \leqslant N \leqslant 100$, using a Metropolis-Hastings algorithm to draw the momentum transfer $q$ in accordance with the rate (15). The initial states $\left|\psi_{0}\right\rangle$ were generated randomly by simplex picking. A $\chi^{2}$-test then confirmed that the asymptotic trajectories, i.e., the pointer states $\mathrm{P}_{i}=\left|\pi_{i}\right\rangle\left\langle\pi_{i}\right|$, are distributed according to $\left|\left\langle\psi_{0} \mid \pi_{i}\right\rangle\right|^{2} \equiv \operatorname{Tr}\left[\rho_{0} \mathrm{P}_{i}\right]$.

This suggests that the solitonic solutions to (7) are the pointer states of collisional decoherence in the sense that they retain their purity, while their superpositions decay into mixtures with weights given by the initial overlap. However, this result was derived under the assumption that the soliton variance is small compared to $2 \pi \hbar^{2} / \operatorname{Var}_{G}(q)$. We will see below that this assumption can be justified for small $\kappa=\sigma_{G}^{2} / m \hbar \gamma$. Still, the finite width leads to a small but finite loss of purity of the solitons, such that Eq. (11) is not an exact equality.

\section{Dynamics and size of the pointer states}

The motion of the pointer states can be characterized by their position and momentum expectation values also in presence of an external potential. Our numerical investigations indicate that the dynamics of this phase space trajectory changes from a quantum behavior to that expected from the corresponding classical mechanics for decreasing position spread $\sigma_{\pi}$ of the pointer states. To explain this fact, we note that 
an additional linear potential $V(x)=a x$ in (7) implies that the solitonic solutions have the form $\pi_{t}(x)=f\left(x-x_{t}\right) e^{i \tilde{\varphi}\left(x-x_{t}, t\right)}$, with $x_{t}=-a t^{2} / 2 m+v_{0} t$ and $f$ still exponentially localized. The pointer states thus move along the accelerated classical trajectories for linear potentials, implying that they will follow the general classical motion if the linearization of the potential is permissible over their spatial extension. Similar observations were made in [7, 21] with linear coupling models.

The pointer state position spread $\sigma_{\pi}$, which depends on the choice of $G(q)$ and $\gamma$, thus serves as an important quantity to characterize the mixture (1). We take $G(q)$ to be a centered Gaussian with variance $\sigma_{G}^{2}$. The dimensionless form of the soliton equation (7) then contains the ratio $\kappa=\sigma_{G}^{2} / m \hbar \gamma$ as the only parameter. By numerically solving (7) we extracted the dimensionless pointer width $\sigma_{\pi} \sigma_{G} / \hbar$ as a function of $\kappa$. We found that the functional form can be reproduced by a simple localization model, which is inspired by studies of collisional decoherence [10, 11, 12] (and easily extended to the 3D case below): one assumes that scattering events occurring with rate $\gamma$ localize the wave function to a length scale $\ell_{\mathrm{loc}}$, characterized by $1-F\left(\ell_{\text {loc }}\right) / F(\infty)=: \exp \left(-a_{\text {loc }}^{2} / 2\right)$, while it disperses freely between the collisions. Averaging the wave function width over the waiting time distribution of a Poissonian process then yields

$$
\sigma_{\pi} \frac{\sigma_{G}}{\hbar}=\frac{\kappa}{4 a_{\mathrm{loc}}}+a_{\mathrm{loc}} .
$$

A value of $a_{\mathrm{loc}} \simeq 0.4$ reproduces the numerically obtained widths very well (better than $10 \%$ ) over the full range of $\kappa$. It follows from (18) that for small $\kappa \ll 4 a_{\mathrm{loc}}^{2}$, the soliton width is given by $\sigma_{\pi}^{2} \simeq a_{\mathrm{loc}}^{2} \hbar^{2} / \sigma_{G}^{2}$, such that the assumption of small position variance of $\phi_{i}(x)$ can be justified for small $\kappa$. In particular, we have checked numerically the approximation $\int \mathrm{d} x e^{i q x / \hbar}\left|\phi_{i}(x)\right|^{2} \simeq e^{i q x_{i} / \hbar}$, by using the solitonic solution of (7); the relative error is less than $2 \%$ for $q \in\left[-2 \sigma_{G}, 2 \sigma_{G}\right]$ and $\kappa \leqslant 10^{-3}$.

\section{Extension to 3D}

Equations (11)-(15) are trivially extended to the 3D situation, though their numerical treatment is then more difficult. However, the above localization model allows one to directly estimate the 3D pointer width, and thus the coherence length of an interacting gas, using the microscopically realistic localization rate $F$ derived in [12. We illustrate this for the case of hard-sphere s-wave scattering off a thermal gas, characterized by the thermal wave length $\Lambda_{\mathrm{th}}=\sqrt{2 \pi / m k_{\mathrm{B}} T} \hbar$. In this case, the localization scale $\xi_{\text {loc }} \equiv \ell_{\text {loc }} / \Lambda_{\text {th }}$ is determined by $\xi_{\text {loc }}=\exp \left(a_{\text {loc }}^{2} / 2-4 \pi \xi_{\text {loc }}^{2}\right) \operatorname{erfi}\left(2 \sqrt{\pi} \xi_{\text {loc }}\right) / 4$, which implies $\xi_{\text {loc }} \simeq 0.1$ if we assume $a_{\text {loc }}=0.4$, as above. The average over the waiting time then yields the width of the pointer state

$$
\sigma_{\pi}=\frac{\ell_{\text {free }}}{16 \xi_{\text {loc }}}+\xi_{\text {loc }} \Lambda_{\text {th }}
$$

where $\ell_{\text {free }}$ is the mean free path. For a weakly interacting or thin gas, the pointer state width is thus essentially determined by $\ell_{\text {free }}$. In the limit of a strongly interacting or dense gas, on the other hand, it is bounded by the scale of the thermal wave length $\Lambda_{\text {th }}$.

We can use (11) to characterize the thermal state of a particle in presence of the interacting gas: the pointer state velocities display a Maxwell distribution, parametrized by $\Lambda_{\mathrm{th}}$, while their position spread is given by (19). Identifying this state with the reduced single particle gas state $\rho_{\text {gas }}$, we can thus access the coherence 
length $\Lambda_{\text {coh }}$ of the self-interacting gas. Since the latter is defined by the decay of the position off-diagonal elements, $\left\langle\boldsymbol{x}\left|\rho_{\text {gas }}\right| \boldsymbol{x}^{\prime}\right\rangle \propto \exp \left(-\pi\left|\boldsymbol{x}-\boldsymbol{x}^{\prime}\right|^{2} / \Lambda_{\text {coh }}^{2}\right)$, one obtains

$$
\frac{1}{\Lambda_{\mathrm{coh}}^{2}}=\frac{1}{\Lambda_{\mathrm{th}}^{2}}+\frac{1}{8 \pi \sigma_{\pi}^{2}}
$$

with $\sigma_{\pi}$ from (19) involving the mean free path. This shows how the interactions in the gas reduce its ideal coherence length, a behavior possibly observable in the interference of cold, non-degenerate gas clouds [22].

\section{Conclusion}

We have shown that the pointer basis of the collisional decoherence master equation is naturally obtained from the orthogonal unraveling, explaining the formation and dynamics of the pointer states, as well as the decay of an initial superposition into the correct mixture. Since collisional decoherence is a paradigm for the non-perturbative description of environmental influences, one may expect this unraveling to generically provide the pointer basis. Apart from its conceptual importance, it also provides an efficient numerical treatment of open quantum dynamics, because the quantum trajectories cease to jump after the decoherence time, leaving the deterministic motion of the pointer states as the only remaining dynamics.

We thank B. Vacchini for helpful discussions. The work was supported by the DFG Emmy Noether program.

[1] W. H. Zurek, Phys. Rev. D 24, 1516 (1981); 26, 1862 (1982); Rev. Mod. Phys. 75, 715 (2003).

[2] E. Joos et al., Decoherence and the Appearance of a Classical World in Quantum Theory (Springer, Berlin, 2003).

[3] M. Schlosshauer, Decoherence and the Quantum-To-Classical Transition (Springer, Berlin, 2007).

[4] W. H. Zurek, S. Habib, and J. P. Paz, Phys. Rev. Lett. 70, 1187 (1993).

[5] L. Diósi, Phys. Lett. A 122, 221 (1987).

[6] N. Gisin and M. Rigo, J. Phys. A: Math. Gen. 28, 7375 (1995).

[7] L. Diósi and C. Kiefer, Phys. Rev. Lett. 85, 3552 (2000).

[8] W. T. Strunz, Lect. Notes Phys. 611, 199 (2002).

[9] J. Eisert, Phys. Rev. Lett. 92, 210401 (2004).

[10] K. Hornberger, S. Uttenthaler, B. Brezger, L. Hackermüller, M. Arndt, and A. Zeilinger, Phys. Rev. Lett. 90, 160401 (2003).

[11] M. R. Gallis and G. N. Fleming, Phys. Rev. A 42, 38 (1990).

[12] K. Hornberger and J. E. Sipe, Phys. Rev. A 68, 012105 (2003).

[13] L. Diósi, Phys. Lett. 114A, 451 (1986).

[14] B. Vacchini, Phys. Rev. Lett. 95, 230402 (2005).

[15] K. Hornberger, Phys. Rev. Lett. 97, 060601 (2006); K. Hornberger and B. Vacchini, Phys. Rev. A 77, 022112 (2008).

[16] J. Klauder and B. Skagerstam, J. Phys. A: Math. Gen. 40, 2093 (2007).

[17] C. W. Gardiner et al., Phys. Rev. A 46, 4363 (1992); H. Carmichael, An Open Systems Approach to Quantum Optics (Springer, Berlin, 1993); K. Mølmer et al., J. Opt. Soc. Am. B 10, 524 (1993).

[18] H.-P. Breuer and F. Petruccione, The Theory of Open Quantum Systems (Oxford University Press, 2007).

[19] L. Diósi, J. Phys. A 21, 2885 (1988).

[20] M. Rigo and N. Gisin, Quantum Semiclass. Opt. 8, 255 (1996).

[21] T. Bhattacharya, S. Habib, and K. Jacobs, Phys. Rev. Lett. 85, 4852 (2000).

[22] D. E. Miller et al., Phys. Rev. A 71, 043615 (2005). 